\title{
Status of the large area MCP-PMT in China
}

\author{
Feng Gao, Sen Qian' ${ }^{\dagger}$, Shulin Liu, Zhe Ning, Yifang Wang, Tianchi Zhao, Yuekun \\ Heng, \\ Institute of High Energy Physics, Chinese Academy of Sciences \\ Beijing 100049, China \\ E-mail: qianseihep.ac.cn
}

\section{Hulin Liu, Weihua Li, Jinshou Tian, Yonglin Wei, Liwei Xin}

$X i$ 'an Institute of Optics and Precision Mechanics, Chinese Academy of Sciences

$X i$ 'an 710068, China

Guorui Huang, Dong Li, Ling Ren, Jianning Sun, Shuguang Si

Nanjing, North Night Vision Tech. Ltd.

Nanjing 211106, China

\section{Ming Qi}

Department of Physics, Nanjing University

Nanjing 210093, China

On behalf of the MCP-PMT workgroup

\begin{abstract}
For the next generation neutrino experiment, number of experienced researchers and engineers in research institutes and companies related to PMT fabrication in China jointly worked on the large area MCP-PMT collaboration group. In the past 5 years by collaborative work, the 8 -inch and 20 -inch prototypes were produced. And also get the high detection efficiency 20 -inch MCP-PMT for JUNO in 2015, and get the $75 \%$ order of the JUNO-PMT. This manuscript will introduce the progress of the R\&D of this new design of the MCP-PMT, also the performance tested result in the PMT Lab in IHEP.
\end{abstract}

38th International Conference on High Energy Physics

3-10 August 2016

Chicago, USA

\footnotetext{
${ }^{1}$ Speaker

+Corresponding author. qians@ihep.ac.cn;

$\boldsymbol{V}$ Copyright owned by the author(s) under the terms of the Creative Commons
Attribution-NonCommercial-NoDerivatives 4.0 International License (CC BY-NC-ND 4.0). http://pos.sissa.it/
} 


\section{Introduction}

Researchers in the Institute of High Energy Physics (IHEP) have conceived a new concept of photomultiplier tube (PMT) for the next generation neutrino experiment in 2009[1]. The small Micro-channel Plate (MCP) units replace the bulky Dynode chain in the traditional large PMTs, called as MCP-PMT. In addition transmission photocathode on the front hemisphere and reflection photocathode on the back hemisphere are fabricated in the same glass bulb to form nearly $4 \pi$ effective photocathode in order to enhance the efficiency of photoelectron conversion, as shown in figure 1 .

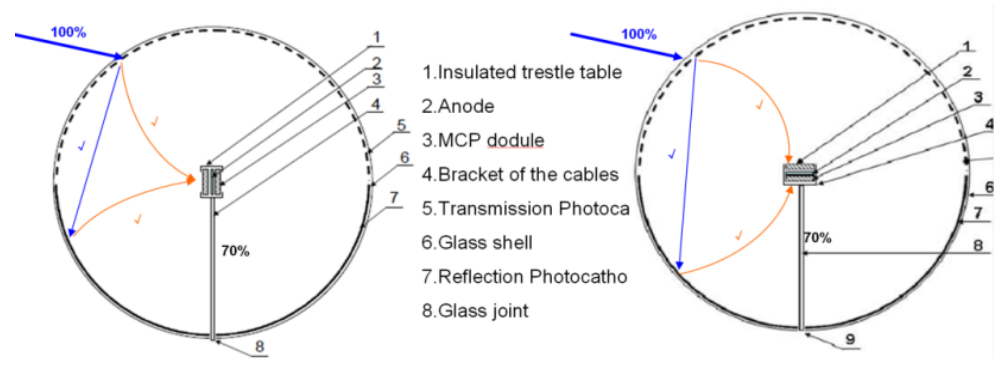

Fig. 1. The conceptual drawing of new large area MCP-PMT

A number of experienced researchers and engineers in research institutes and companies related to PMT fabrication in China jointly worked on the large area MCP-PMT project from 2011. After several years R\&D, a number of 8-inch prototypes were produced in 2012 but can not test the single photoelectron spectrum (SPE), only with number quantum efficiency (QE) about 25\%@410nm.

Then, at the end of 2013, the 8-inch MCP-PMT prototypes could with the peak-to valley ratio $(\mathrm{P} / \mathrm{V})$ of single photoelectron spectrum $(\mathrm{SPE})>2.0$ and $\mathrm{QE} \sim 25 \% @ 410 \mathrm{~nm}$. Also the other performances were carefully tested by the PMT evaluation system in the PMT Lab in IHEP [2], and the test data were shown in table.1.

In 2013, a generic underground national lab for neutrino physics was lead by the IHEP, and under construction in Jiangmen, Guangdong province in south China. The Jiangmen Underground Neutrino Observatory (JUNO) requires a high performance large detector, which employs approximately 20,000 pieces of 20-inch PMTs that have large sensitive area, high quantum efficiency, high gain and large $\mathrm{P} / \mathrm{V}$ value for good photon counting performance [3].

So, the MCP-PMT collaboration group decided to produce the 20-inch MCP-PMT for JUNO. And the North Night Vision Tech. Ltd. (NNVT) in Nanjing, China, one of the members of the MCP-PMT collaboration group has produced this type of 20-inch MCP-PMT in 2014.

The performance of this type of 20-inch PMTs, the QE, the P/V of the SPE, the collection efficiency (CE), the transit time spread (TTS), Rise time / fall time of the waveform, the anode dark rate, et.al were also carefully tested in the PMT Lab in IHEP as shown in table.1.

Table 1. Characterists of the 8-inch and 20-inch MCP-PMTs

\begin{tabular}{|c|c|c|c|c|c|c|c|c|}
\hline Items & $\begin{array}{c}\text { QE @ } \\
410 \mathrm{~nm}\end{array}$ & CE & SPE P/V & TTS & $\begin{array}{c}\text { Rise } \\
\text { time }\end{array}$ & Fall time & $\begin{array}{c}\text { Anode } \\
\text { dark rate }\end{array}$ & $\begin{array}{c}\text { After } \\
\text { pulse rate }\end{array}$ \\
\hline Units & $\%$ & $\%$ & & $\mathrm{~ns}$ & $\mathrm{~ns}$ & $\mathrm{~ns}$ & $\mathrm{~Hz}$ & $\%$ \\
\hline $\mathbf{2 0}$ inch & 25 & $70 \%$ & $>3$ & $\sim 12$ & 2 & 10 & $\sim 30 \mathrm{k}$ & 3 \\
\hline $\mathbf{8}$ inch & 25 & $70 \%$ & $>2$ & $\sim 3$ & 5 & 6 & $\sim 2.2 \mathrm{k}$ & small \\
\hline
\end{tabular}




\section{The Quantum Efficiency of the MCP-PMTs}

The area coverage and the quantum efficiency (QE) of the PMT photocathode are critical in such experiments in order to capture as much signal light as possible from neutrino reactions in the detection media. Details of the QE experiments and corresponding results of MCP-PMTs will be presented in this paper.

To measure the QE of the photocathode, the cathode current should be measured carefully by the electrometer. A photocathode current at the monochromatic light (@410nm)versus voltage curve (I-V curve) as shown in figure 2 was measured first. The current plateau indicates that all photoelectrons emitted from the cathode are collected by the electrod, thus, the corresponding current value should be adopted in the QE calculation. The QE studied by measuring the I-V curves with a light spot illuminating the photocathode top center are shown in figure 2. the results show the QE of 20-inch MCP-PMT 78\# are 23.25\% @400nm.

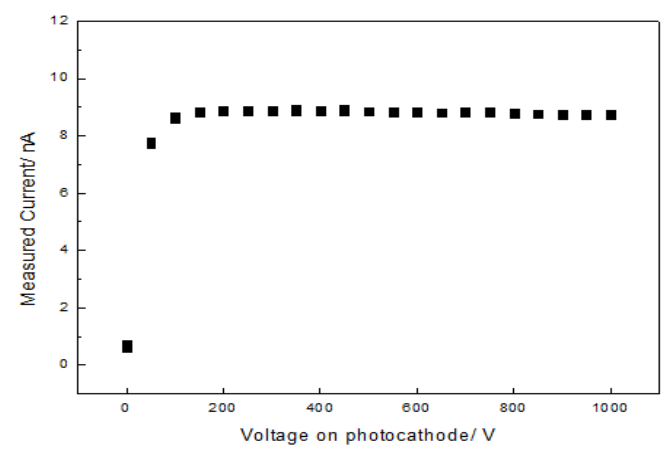

Figure 2. Measured current versus voltage curves for MCP-PMT @400nm:I-V

A tungsten-halogen lamp and a monochromator were utilized as the light source in the photocathode spectral response measurements. Monochromatic lights of $3 \mathrm{~nm}$ width were used in $10 \mathrm{~nm}$ steps from $300 \mathrm{~nm}$ to $700 \mathrm{~nm}$ to measure the QE at each corresponding wavelength.

The QE spectral response of one 20-inch MCP-PMT was shown in figure 3. The results indicate that QE value reaches a peak response around 390nm with a light spot illuminating the photocathode top center. And this MCP-PMT 78\# achieves a peak QE value of 23.53\%@410nm as the same data as shown in figure 2 . The short different is mainly of the uniformity of large area photocathode in 20-inch MCP-PMT.

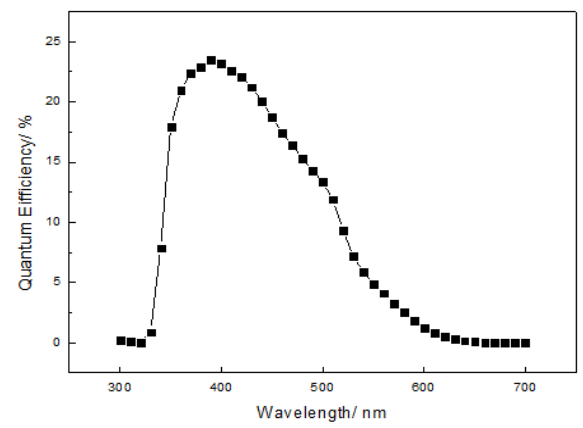

Figure 3. Quantum efficiency versus wavelength for MCP-PMT: QE-wavelength (nm)

The measurement data of the QE is different when the photocathode receive a beam of same incident light with different path. There are four incident paths as shown in figure4. Single transmission(a), two transmission(b), vertical incidence to reflection of aluminium(c), oblique incidence to reflection of aluminium (d). Table 2 lists the measured QE in the differentd incident path using a same light at $410 \mathrm{~nm}$. 

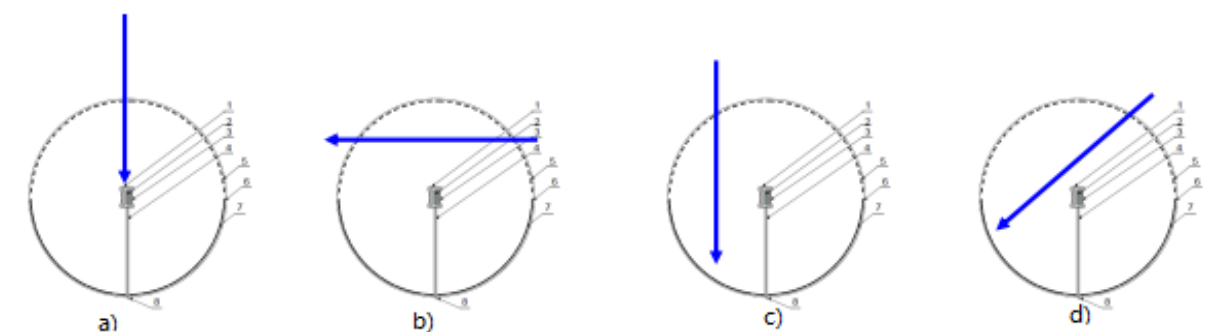

Figure 4. The different path of same incident light through photocathode of the 20-inch MCP-PMT

Table 2. The QE in the different mode photocathodes@410nm

\begin{tabular}{|c|c|c|c|c|}
\hline Mode & $\mathrm{a}$ & $\mathrm{b}$ & $\mathrm{c}$ & $\mathrm{d}$ \\
\hline $78 \#$ & $23.25 \%$ & $26.98 \%$ & $27.97 \%$ & $31.55 \%$ \\
\hline
\end{tabular}

As the design of the MCP-PMT, the addition transmission photocathode on the front hemisphere and reflection photocathode on the rare hemisphere are fabricated in the same glass bulb to enhance the efficiency of photoelectron conversion. The photocathode coverage of this type of MCP-PMT is larger than the usual dynode-PMT, so it will capture as much signal light as possible from neutrino reactions in the detection media.

\section{The High Detection Efficiency MCP-PMTs}

In 2015, people in the collaboration group had get great progress for to improving the performance of the 20-inch MCP-PMT prototypes, especially the collection efficiency of the MCP [4]. For the primary prototypes, the CE of the MCP was depend on the open area ratio of the MCP $\sim 70 \%$. With some special technology about the MCPs, to change the depth of the output electrode, the diameter of the MCP, the diameter of the channels, the inclined angle of the channels, and so on.

Finally, at the middle of 2015, the new 20-inch MCP-PMT prototype with high detection efficiency (HDE) was produced. This design was changed compared with the primary ones, only using one MCPs module at the downward part of the prototype to collect all the photoelectron from both the transmission photocathode and reflection photocathode, as shown in figure 5 .

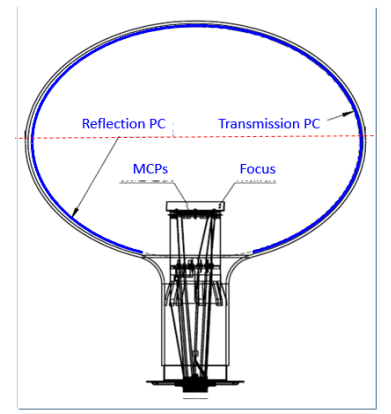

Fig. 5 The new typical prototypes of the 20 inch HDE MCP-PMT ßå

The characteristics of the 20-inch HDE-MCP-PMT were carefully studied by measuring the photocathode (Trans. PC), such as I-V curves, the quantum efficiency (QE) vs. wavelength, and the mapping of the QE uniformity. Also the SPE, the timing properties of anode signals, the anode linearity, the noise characteristics, the after pulse properties were studied at gain 
$\sim 1.0 \times 10^{7}$. The main parameters could be seen in table.3, the primary MCP-PMT with $70 \% \mathrm{CE}$, the HDE, and also one prototype with dynode part.

Table 3. Comparison of the 20 inch PMTs

\begin{tabular}{|c|c|c|c|c|}
\hline Items & Units & Pri-MCP-PMT & HDE-MCP-PMT & R12860 \\
\hline Size & inch & $\mathbf{2 0}$ & $\mathbf{2 0}$ & $\mathbf{2 0}$ \\
\hline QE at $410 \mathrm{~nm}$ & $\%$ & $\mathbf{2 5}$ & $\mathbf{2 6}$ & $\mathbf{3 0}$ \\
\hline Relativity CE & $\%$ & $\mathbf{7 0}$ & $\mathbf{1 0 0}$ & $\mathbf{9 0 ?}$ \\
\hline Relativity DE & $\%$ & $\mathbf{6 0 \%}$ & $\sim \mathbf{1 1 0} \%$ & $\mathbf{1 0 0 \%}$ \\
\hline SPE P/V & & $>\mathbf{2}$ & $>\mathbf{3}$ & $>\mathbf{3}$ \\
\hline TTS (top point) & $\mathrm{ns}$ & $\sim \mathbf{1 0}$ & $\sim \mathbf{1 2}$ & $\sim \mathbf{3}$ \\
\hline Rise time & $\mathrm{ns}$ & $\mathbf{2}$ & $\mathbf{2}$ & $\mathbf{7}$ \\
\hline Fall time & $\mathrm{ns}$ & $\mathbf{1 0}$ & $\mathbf{1 0}$ & $\mathbf{1 7}$ \\
\hline Anode dark rate & $\mathrm{Hz}$ & $\sim \mathbf{3 0} \mathbf{k}$ & $\sim \mathbf{3 0} \mathbf{k}$ & $\sim \mathbf{3 0} \mathbf{k}$ \\
\hline After pulses time delay & $\mu \mathrm{s}$ & $/$ & $\sim \mathbf{4 . 5}$ & $\sim 4$ and $\sim 17$ \\
\hline After pulse rate & $\%$ & small & $\mathbf{3}$ & 10 \\
\hline Radiation background & & high & $\mathbf{l o w}$ & high \\
\hline
\end{tabular}

With the large area about the photocathode, the QE and DE will be improved, but the TTS and dark noise will be worse. So, the users need to get the balance between these above parameters for different physics aims.

Especially, the glass used for 20 inch MCP-PMT has extra low potassium, low uranium and the contents resulting extra low radiation background. The PMT purchase of JUNO

The JUNO Bidding started on Oct.23th 2015, and completed on Nov.17th 2015. Compensating the PMT performance with fiducially volume convert all specifications to cost, radioactivity, dark noise, TTS, and so on in table.3, the JUNO ordered 15000 pic 20 inch MCPPMT from the NNVT.

\section{Acknowledgments}

The MCP-PMT development project has been partially supported by the Strategic Priority Research Program of the Chinese Academy of Sciences (No. XDA10010400) and the National Natural Science Foundation of China (Grant No.11175198, No.11475209 and No.11675205).

\section{References}

1. Yifang Wang, et al., Nuclear Instruments and Methods A 695 (2012) 113.

2. J. Xia, A performance evaluation system for photomultiplier tubes, JINST 10 P03023, 2015.

3. Yu-Feng Li,et, al., Unambiguous determination of the neutrino mass hierarchy using reactor neutrinos, Physical Review D 88, 013008(2013).

4. Yaping Chang, et al., The R\&D of the 20 inch MCP-PMTs for JUNO, Nucl. Instr. and Meth, A 824 (2016) 143-144 